\title{
HER2 Amplification in Advanced NSCLC Patients After Progression on EGFR-TKI and Clinical Response to EGFR-TKI Plus Pyrotinib Combination Therapy
}

Jiadi Gan

Yihua Huang

Jun Liao

Lanlan Pang

Wenfeng Fang

Department of Medical Oncology, State Key Laboratory of Oncology in South China, Collaborative Innovation Center for Cancer Medicine, Sun Yat-sen University Cancer Center, Guangzhou, 510060, People's Republic of China
Correspondence: Wenfeng Fang Department of Medical Oncology, State Key Laboratory of Oncology in South China, Collaborative Innovation Center for Cancer Medicine, Sun Yat-sen University Cancer Center, Guangzhou, 510060, Peoples Republic of China Email fangwf@sysucc.org.cn
Background: HER2 (or ERBB2) amplification is an important mechanism for acquired resistance to EGFR tyrosine kinase inhibitors (TKI). The benefits of HER2-targeted therapy have been limited. Herein, we investigated the molecular and clinical patterns of HER2 amplification in non-small cell lung cancer (NSCLC) patients during progression on EGFRTKIs and the potential of combining EGFR-TKI and pyrotinib to overcome resistance.

Methods: In this study, 1,637 NSCLC cases from Geneseeq after progression of EGFRTKIs were screened and analyzed by next generation sequencing (NGS), in which 48 patients with HER2 amplification were eligible and enrolled. A total of 403 patients from Sun Yat-sen University Cancer Center (SYSUCC) were screened and five patients with concomitant EGFR mutations and HER2 amplification were retrospectively collected to assess the effect of afatinib or combination of EGFR-TKI and pyrotinib.

Results: In the 48 patients from the Geneseeq cohort, $27(56.2 \%)$ patients suffered from resistance of 1st/2nd generation EGFR-TKI, and 21 (43.8\%) patients from 3rd generation. As for the five patients forming the SYSUCC cohort, three patients were treated with afatinib, one achieved partial response (PR) with progression-free survival (PFS) of 6 months and two quickly developed disease progression. Two patients were treated with EGFR-TKIs plus pyrotinib, one receiving gefitinib plus pyrotinib achieved PR with PFS of 8 months and benefited from osimertinib plus pyrotinib for 3 months till data-off; one receiving osimertinib plus pyrotinib achieved SD for 4 months till data-off. The most common co-occurring alteration was TP53 $(91.7 \%)$ in the mutation profile of the 48 patients from the Geneseeq cohort, and four patients had TP53 co-mutations of the five patients from the SYSUCC cohort.

Conclusion: In this study, we detected 7\% HER2 amplification present in EGFR-TKIs resistance. Patients with concomitant EGFR mutation and HER2 amplification may derive clinical benefit from therapies that target both EGFR and HER2.

Keywords: non-small cell lung cancer, NSCLC, epidermal growth factor receptor tyrosine kinase inhibitors, EGFR TKIs, acquired resistance, combined therapy

\section{Introduction}

Lung cancer has the highest mortality among all malignancies, with NSCLC accounting for approximately $85 \%$ of all lung cancer cases. ${ }^{1}$ Recent progress in the identification of oncogenic driver mutations has triggered the development of novel therapeutic approaches, and the development of molecular-targeting antitumor drugs has improved clinical outcomes in NSCLC patients. For example, 
target therapies for patients with epidermal growth factor receptors (EGFR), anaplastic lymphoma kinase (ALK), ROS proto-oncogene 1 receptor tyrosine kinase (ROS1), and B-Raf proto-oncogene serine/threonine kinase (BRAF) mutations are promising strategies for improving the clinical outcome of patients with NSCLC. ${ }^{2-5}$

Somatic, activating mutations in the EGFR gene are present in about $15 \%$ of Caucasian and nearly $50 \%$ of Asian patients with advanced NSCLC. ${ }^{6,7}$ The firstgeneration tyrosine kinase inhibitor (TKI) gefitinib and erlotinib and second-generation TKI afatinib and dacomitinib were recommended as first-line therapy for advanced EGFR-mutated NSCLC with average PFS ranging from 9 to 15 months. $^{8}$ Osimertinib demonstrated 18.9 months of PFS and was approved in 2018 as first-line therapy for advanced EGFR-mutated NSCLC, regardless of T790M mutation status. ${ }^{9}$ However, the majority of patients inevitably develop resistance including altered drug target, bypass or alternative track activation, and morphological alterations. $^{10}$

The HER2 gene has been identified as a driving gene and potential therapeutic target for lung cancer. ${ }^{11}$ Recently, studies demonstrated HER2 alterations could present concomitantly with EGFR mutation. ${ }^{12}$ Around $10 \%$ of HER2 cases occur in EGFR-TKI resistance. ${ }^{13}$ HER2 amplification is described as one of the major mechanisms of acquired resistance to first generation TKIs in EGFRmutant lung cancers. ${ }^{14}$ HER2 gene amplification and protein overexpression, lacking the corresponding ligand, can directly induce the formation of HER2 homodimers or heterodimers (including EGFR, HER3, HER4) receptor tyrosine kinase and downstream signaling pathways, and promote proliferation and metastasis of tumor cells.

The clinical activity of anti-HER2 agents in lung cancer is limited. Chemotherapy is still the preferred regimen for patients with advanced NSCLC who are improper for targeted therapy. Trastuzumab emtansine (T-DM1) was recommended for treatment of NSCLC by the National Comprehensive Cancer Network (NCCN) guidelines. ${ }^{15}$ Several studies and case series have shown clinical benefit from HER2 inhibitors neratinib, ${ }^{16}$ dacomitinib, ${ }^{17}$ and pyrotinib. ${ }^{18}$ However, lapatinib showed unsatisfactory efficacy in lung cancer harboring HER2 alteration. ${ }^{19}$ Treatment of HER2-positive lung cancer was explored mainly in exon 20 insertion with afatinib and presented promising efficacy. Phase I/II studies of trastuzumab on HER2 amplification/overexpression, TDM-1 on amplification/overexpression, and DS-8201a on overexpression/ mutation showed low-to-moderate efficiency with lung cancer (ORR of 23-46\% for trastuzumab, 20-50\% for TDM-1, and $62.5 \%$ for DS-8201a). ${ }^{20}$ A Phase I/II study of TAK-788 in adults with NSCLC explores the efficacy of TAK-788 in HER2 mutations patients, and the efficacy is needed to be identified in the future (ClinicalTrials.gov identifier: NCT02716116).

The aim of the current study is to determine the frequency and molecular spectrum of HER2 amplification and to take advantage of the available knowledge to preliminarily explore corresponding strategies in a large cohort of patients with lung adenocarcinomas posttreated with EGFR-TKIs. We describe five cases of patients who progressed on $1 \mathrm{st} / 2$ nd or 3rd generation EGFR-TKIs from our center SYSUCC and developed HER2 amplification with EGFR mutation retained but achieved a remarkable response after combination treatment with EGFR-TKI and pyrotinib.

\section{Methods}

\section{Patients}

A total of 1,637 patients from Geneseeq with advanced EGFR-mutant NSCLC treated with 1st/2nd or 3rd generation EGFR-TKIs who underwent a tissue re-biopsy or whole blood extracted after progression between January 2015 and July 2020 were retrospectively screened (Figure 1). Forty-eight patients with concomitant EGFR mutations and HER2 amplification were enrolled. Baseline characteristics including age, sex, type of histologic, prior chemotherapy, prior EGFR-TKI therapy, treatment lines, and sample type for NGS were obtained through medical records (Table 1 and Supplementary Table S1). Five NSCLC patients treated with afatinib or a combination of EGFR-TKI and pyrotinib are listed in the Table 2 with the order of 1 to 5. Besides, 403 patients from SYSUCC were screened, 14 patients with concomitant EGFR mutations and HER2 amplification were enrolled, of whom three patients were assigned to other targeted therapy, three patients were assigned to chemotherapy, and two patients were lost to follow-up. The treatment process and concise mutation profile of postEGFR-TKI treatment of these 14 patients is shown in Table 2. The whole mutation profile of pre- and post-EGFRTKI treatment of the 14 patients is shown in Supplementary Table S2. Finally, five patients who received afatinib or a combination of EGFR-TKI and pyrotinib were retrospectively enrolled (Figure 2). All patients signed informed consent forms for treatment in this protocol. 

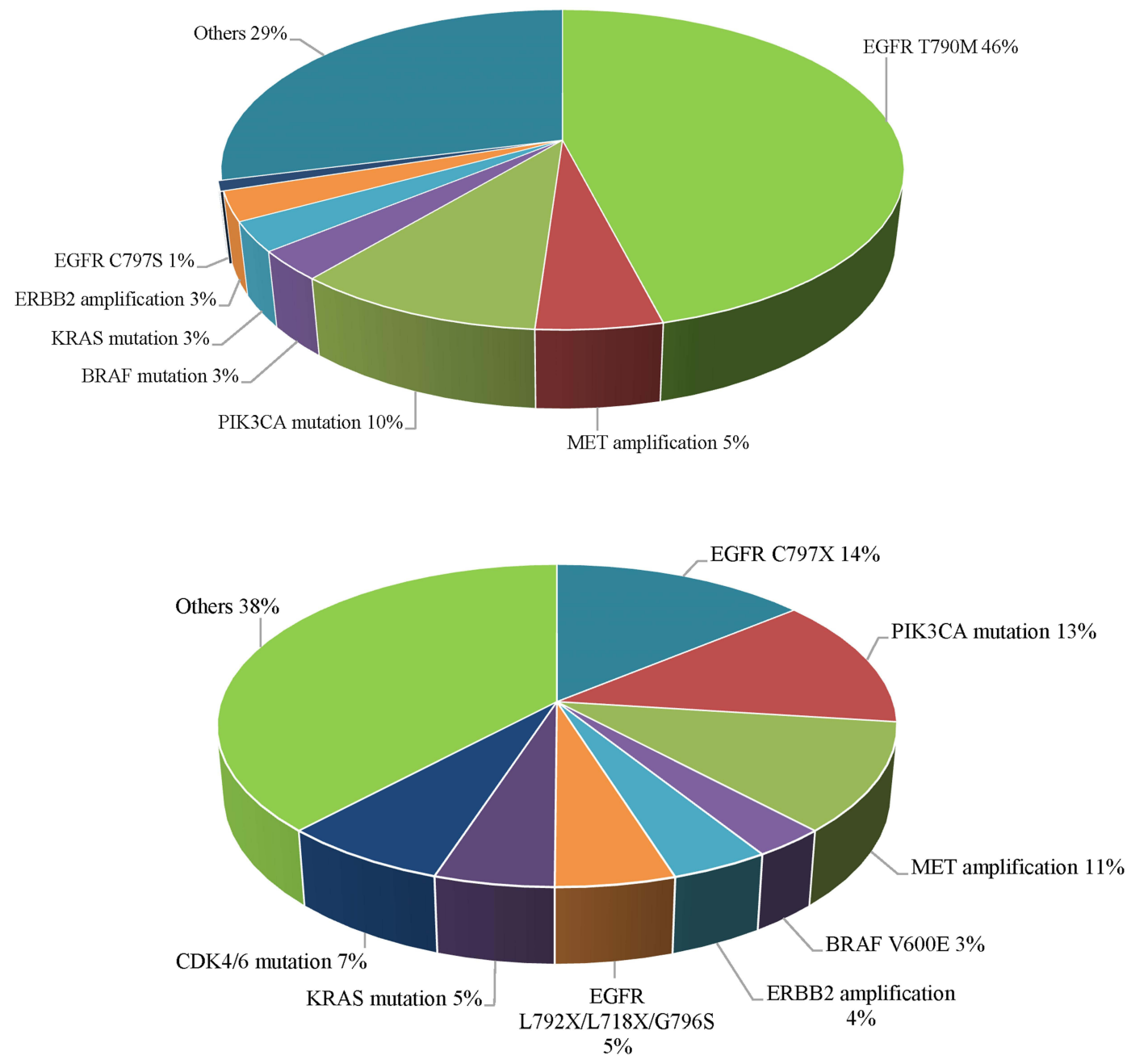

Figure I The various drug resistance mechanisms found in patients with EGFR-TKIs-treated NSCLC from the Geneseeq cohort.

This study was conducted in accordance with the Declaration of Helsinki (as revised in 2013) and approved by Sun Yat-sen University Cancer Center IRB (B2018092-01).

\section{Genetic Analysis}

DNA was extracted using the DNeasy Blood \& Tissue Kit (Qiagen, Hilden, Germany. Purified DNA was qualified by Nanodrop One (Thermo Fisher, Waltham, MA) and quantified by Qubit 3.0 (Life Technologies, Singapore) using the dsDNA HS Assay Kit (Life Technologies, Eugene, OR) according to the manufacturer's recommendations. Different libraries with unique indices were pooled together in desirable ratios for up to $2 \mu \mathrm{g}$ of total library input. Human cot-1 DNA (Life Technologies, Carlsbad, CA) and xGen Universal blocking oligos (Integrated DNA Technologies, Coralville, IA) were added as blocking reagents. The capture reaction was performed with the NimbleGen SeqCap EZ
Hybridization and Wash Kit (Roche, Madison, WI) and Dynabeads M-270 (Life Technologies, Vilnius, Lithuania) with optimized manufacturers' protocols. Captured libraries were on-beads amplified with Illumina $\mathrm{p} 5$ and $\mathrm{p} 7$ primers in KAPA HiFi HotStart ReadyMix (KAPA Biosystems, Cape Town, South Africa). The post-capture amplified library was purified by Agencourt AMPure XP beads and quantified by qPCR using the KAPA Library Quantification kit (KAPA Biosystems). Library fragment size was determined by the Agilent Technologies 2100 Bioanalyzer (Agilent, Santa Clara, CA). The target-enriched library was then sequenced on HiSeq NGS platforms (Illumina, San Diego, CA) according to the manufacturer's instructions.

\section{Outcomes}

Five post-EGFR-TKI treated NSCLC patients with concomitant EGFR mutations and HER2 amplification were enrolled to treat with afatinib monotherapy or 
Table I Clinical Characteristics of the 48 Patients with Concomitant EGFR Mutations and HER2 Amplification Lung Cancer

\begin{tabular}{|l|c|}
\hline Characteristic & Patients \\
\hline Age, years, median (range) & $56.5(39-84)$ \\
\hline Sex, $\mathrm{n}(\%)$ & \\
Male & $21(43.8 \%)$ \\
Female & $27(56.2 \%)$ \\
\hline Histology, n (\%) & \\
Adenocarcinoma & $45(93.7 \%)$ \\
Adenocarcinoma with mixed SCLC & $1(2.1 \%)$ \\
Large cell neuroendocrine carcinoma & $1(2.1 \%)$ \\
Unknown & $1(2.1 \%)$ \\
\hline Prior chemotherapy, $\mathrm{n}(\%)$ & \\
Yes & $12(25 \%)$ \\
No & $33(68.8 \%)$ \\
Unknown & $3(6.2 \%)$ \\
\hline EGFR-TKI resistance, $\mathrm{n}(\%)$ & \\
Resistance to Ist/2nd generation & $27(56.2 \%)$ \\
Resistance to 3rd generation & $21(43.8 \%)$ \\
\hline Treatment lines, $\mathrm{n}$ (\%) & $28(41.7 \%)$ \\
Post-Ist/2nd line treatment & $31(64.6 \%)$ \\
Post-3rd/3rd line post-treatment & $17(35.4 \%)$ \\
\hline Sample type for NGS, $\mathrm{n}(\%)$ & \\
Tumor tissue & \\
ctDNA & \\
\hline
\end{tabular}

a combination of EGFR-TKI and pyrotinib. Computed tomography (CT) on chest and abdomen were routinely performed at the first month after initiation of therapy and then 3 months later. Magnetic Resonance Imaging (MRI) of the brain was required only in patients with known or suspected central nervous system (CNS) metastases. The clinical response was confirmed according to RECIST v1.1, as determined by the investigator. Complete response (CR), partial response (PR), and stable disease (SD) were determined on two consecutive assessments 4 weeks apart. ${ }^{21}$ The assessment of toxicity was dependent on the Common Terminology Criteria for Adverse Events (CTCAE) 4.0.

\section{Results}

\section{Clinical Characteristics of the 48 Patients Enrolled}

The clinical characteristics of the 48 patients enrolled resistant to EGFR-TKIs are summarized in Table 1.
Median age of HER2 amplification patients was 56.5 years (ranging from 39 to 84 years), and the majority of patients were female (56.2\%). Adenocarcinoma was the predominant histology type (93.7\%), and there was one (2.1\%) adenocarcinoma with mixed SCLC, one $(2.1 \%)$ large cell neuroendocrine carcinoma, and the histology type of one patient $(2.1 \%)$ was not available. Twentyseven $(56.2 \%)$ patients suffered from resistance of 1 st/ 2nd generation EGFR-TKI, and 21 (43.8\%) patients from 3 rd generation. Twelve (25\%) patients received prior chemotherapy, $33(68.8 \%)$ patients did not, and the status of prior-chemotherapy was not available for three (6.2\%) patients. Thirty-one (64.6\%) patients suffered from 1 st/ 2nd line treatment and 17 from $3 \mathrm{rd} / 3 \mathrm{rd}$ post-line treatment. Genetic status was based on archival tissue samples or freshly obtained tissue samples (collected within 2 weeks before the start of study treatment) for 20 patients $(41.7 \%)$, and blood samples for 28 patients (58.3\%).

As shown in Figure 3, the 48 lung cancer patients enrolled also harbored other concomitant mutations in NGS results, of which 44 (91.7\%) patients had TP53 comutations and makes the most common one.

\section{Outcomes of Afatinib Treatment in Patients with Concomitant EGFR Mutations and HER2 Amplification Lung Adenocarcinomas}

\section{Patient I}

The first patient was a 47-year-old woman with a recurrent coughing, who presented to the local hospital in March 2019. Chest computed tomography (CT) scan revealed a space-occupying lesion in the right lower lobe with bilateral lung, right lobe of liver, and left adrenal gland metastasis. The pathologic examination by ultrasound guided biopsy results were consistent with moderately differentiated adenocarcinoma of the lung primary. She was treated with one cycle of paclitaxel-albumin +carboplatin without timely NGS since her heavy condition of disease. The pretreatment tissue sample for targeted NGS for 520 cancer-relevant genes revealed EGFR L858R/T790M double mutation. The patient then received osimertinib for 1 month but her disease was been alleviated. NGS of re-biopsy presented a novel HER2 amplification with EGFR L858R/T790M double mutation reserved. She was then treated with afatinib. Unfortunately, her illness continued to deteriorate and she died quite quickly. 


\begin{tabular}{|c|c|c|c|c|c|c|c|c|c|c|c|c|c|}
\hline 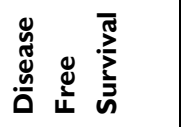 & $\underline{\varepsilon}$ & $\underline{\mathrm{E}}$ & & $\xi$ & & $\underset{f}{\xi}$ & $\underset{\infty}{E}$ & $\underset{f}{\xi}$ & ह & $\S$ & $\underline{\text { ป }}$ & $\S$ & $\S$ \\
\hline 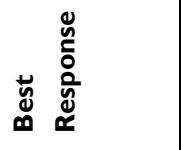 & $\S$ & $\stackrel{\mathscr{O}}{\alpha}$ & & $\stackrel{\alpha}{\alpha}$ & & 只 & $\frac{\alpha}{\alpha}$ & $\stackrel{\alpha}{0}$ & 只 & $\S$ & 只 & $\S$ & $\S$ \\
\hline 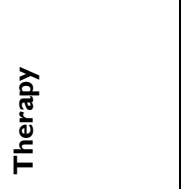 & 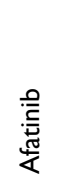 & 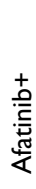 & 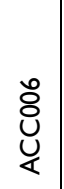 & 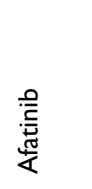 & & 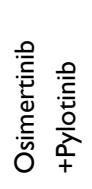 & 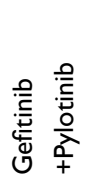 & 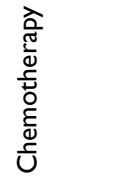 & 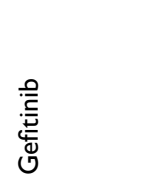 & $\mathbb{z}$ & 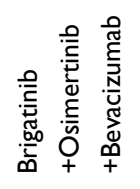 & $\S$ & $\S$ \\
\hline 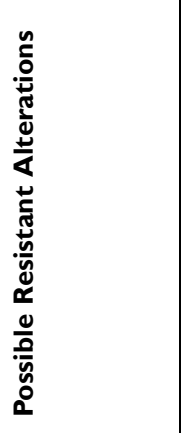 & 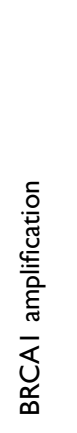 & & 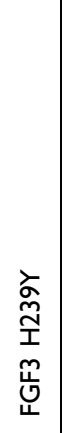 & $\overline{\mathbb{4}}$ & 흠 & $\S$ & 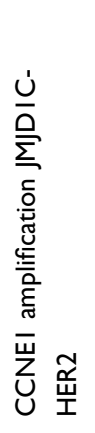 & 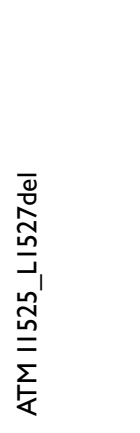 & 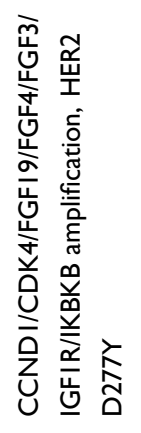 & 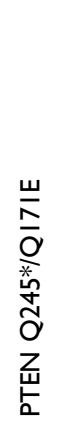 & 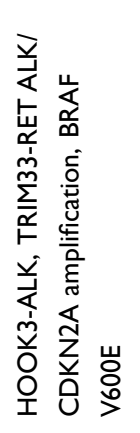 & $\mathbb{z}$ & 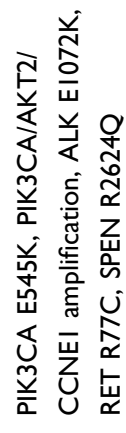 \\
\hline 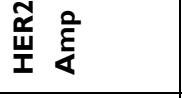 & + & + & & + & & + & + & + & + & + & + & + & + \\
\hline 竞 & 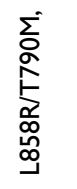 & 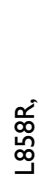 & & 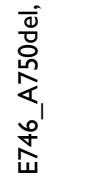 & & 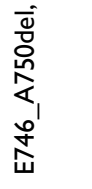 & 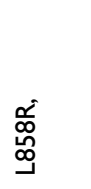 & 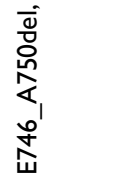 & 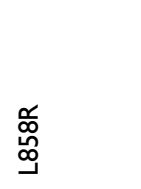 & 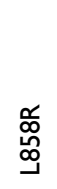 & 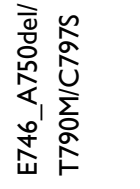 & 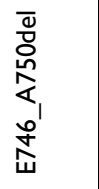 & 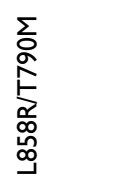 \\
\hline 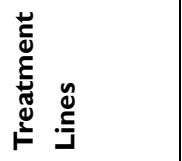 & 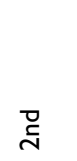 & ָे & & $\ddot{\ddot{n}}$ & & 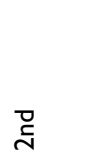 & $\underline{\underline{\Delta}}$ & 点 & $\stackrel{D}{m}$ & $\stackrel{D}{m}$ & $\underset{\sim}{\mathscr{N}}$ & 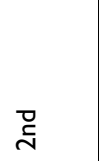 & 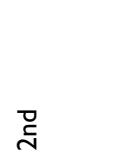 \\
\hline 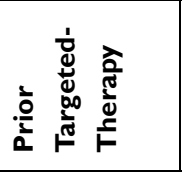 & 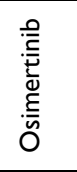 & 黄 & & 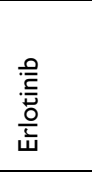 & & 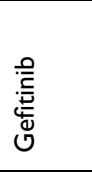 & 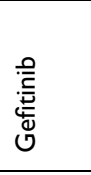 & 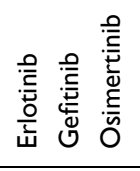 & 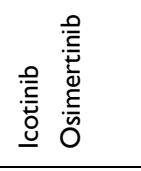 & 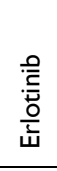 & 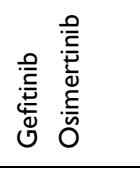 & 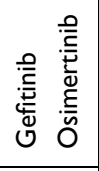 & 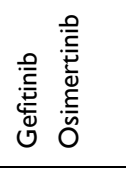 \\
\hline 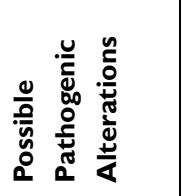 & 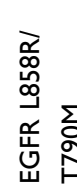 & 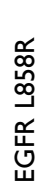 & & 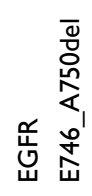 & & 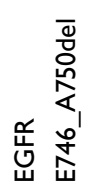 & $\begin{array}{l}\text { ơ } \\
\text { 员 } \\
\text { 品 } \\
\text { 菢 }\end{array}$ & 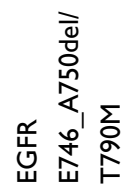 & 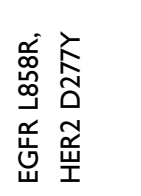 & 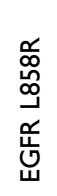 & 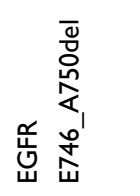 & 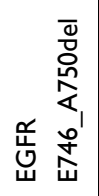 & 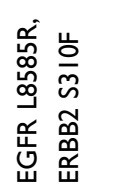 \\
\hline 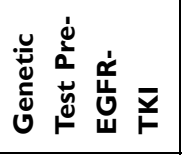 & पू & $\mathbb{Z}$ & & 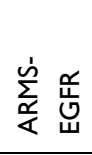 & & $\S$ & క్ & 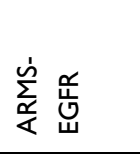 & पू & 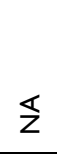 & 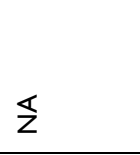 & $\S$ & 气ू \\
\hline$\stackrel{8}{8}$ & $\hat{f}$ & 员 & & $\bar{n}$ & & $\mathscr{f}$ & $\bar{r}$ & in & 요 & மீ & $\pi$ & นก & $\stackrel{\infty}{\infty}$ \\
\hline$\stackrel{x}{凶}$ & ч & $\Sigma$ & & $\Sigma$ & & 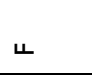 & $\Sigma$ & ч & ч & \llcorner & ч & ч & $\Sigma$ \\
\hline 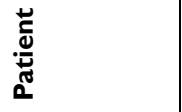 & - & $N$ & & $m$ & & $\sigma$ & in & 0 & $N$ & $\infty$ & $\sigma$ & 으 & $=$ \\
\hline
\end{tabular}




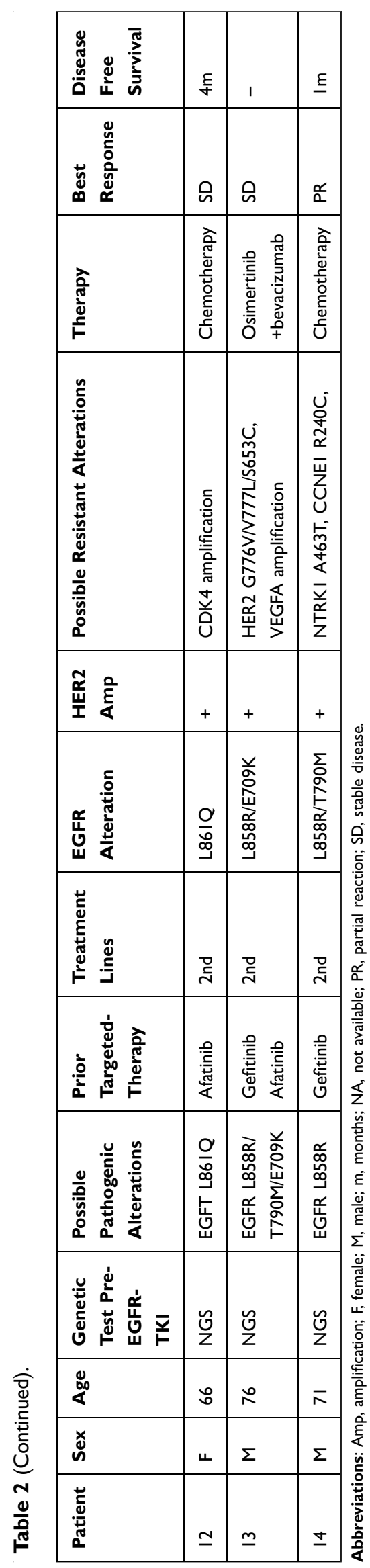

\section{Patient 2}

The second patient was a 54-year-old man with IIIA poorly differentiated lung adenocarcinoma diagnosed in early 2015. He had surgical resection and underwent neoadjuvant chemo-radiotherapy for 4 months. Simultaneously, the patient was treated with gefitinib for 1 year by September 2019. A CT scan demonstrated disease recurrence. Chemotherapy of pemetrexed+cisplatin, pemetrexed, and docetaxel were administered successively for one cycle. A re-test of 508 cancer-relevant genes NGS presented EGFR L858R and HER2 amplification co-alterations. Therefore, he was enrolled in a clinical trial and commenced on afatinib with ACC006 (a novel dual target antitumor drug on both mTOR and hedgehog pathway). However, radiograph evaluation 1 month later indicated progression disease. Subsequently, the patient underwent chemotherapy and immunotherapy and was still alive at study completion.

\section{Patient 3}

The third patient was a 51-year-old man with consecutive cough and scapular pain. The pathological diagnosis was poorly differentiated lung adenocarcinoma and targeted NGS of the tumor biopsy specimen revealed EGFR E746_A750del. He benefited from first-line treatment with erlotinib for PFS of 5 months. A second NGS revealed the presence of concomitant EGFR E746_A750del and HER2 amplification. In view of the known resistance to first generation EGFR-TKI, the patient was treated with afatinib at a dose of $40 \mathrm{mg}$ per day with best efficacy of PR. However, he suffered from emesia and dizziness after 6 months. Brain MRI performed demonstrated multiple intracranial metastatic nodules. The patient then received brain radiotherapy, nevertheless, a repeated MRI 2 month later presented partial increased lesions and some new lesions within the bilateral frontal lobes. The patient was placed on palliative treatment thereafter.

\section{Outcomes of Combined Treatment of} EGFR-TKI and Pyrotinib in Patients with Concomitant EGFR Mutations and HER2 Amplification Lung Adenocarcinomas

\section{Patient 4}

The fourth patient was a 42-year-old woman with paroxysmal cough (Figure 4). Chest-CT presented with a left upper lobe mass with bilateral lung metastasis. MRI 


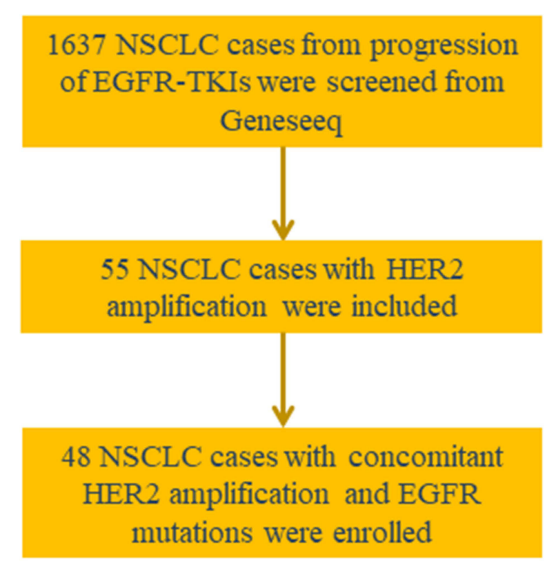

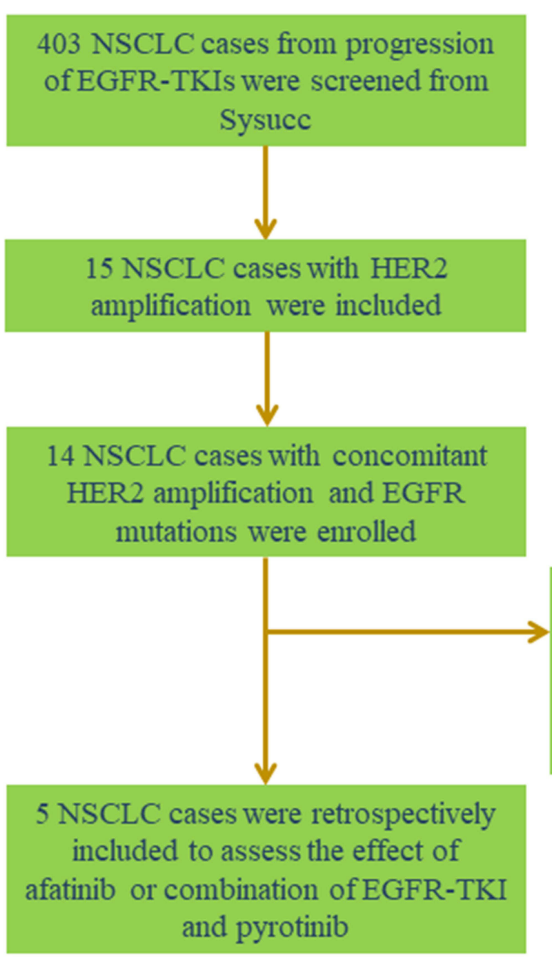

9 NSCLC cases excluded, with reasons: -6 chosen to other targeted therapy -2 chosen to chemotherapy -1 lost to follow up

Figure 2 Patient flow of the study design.

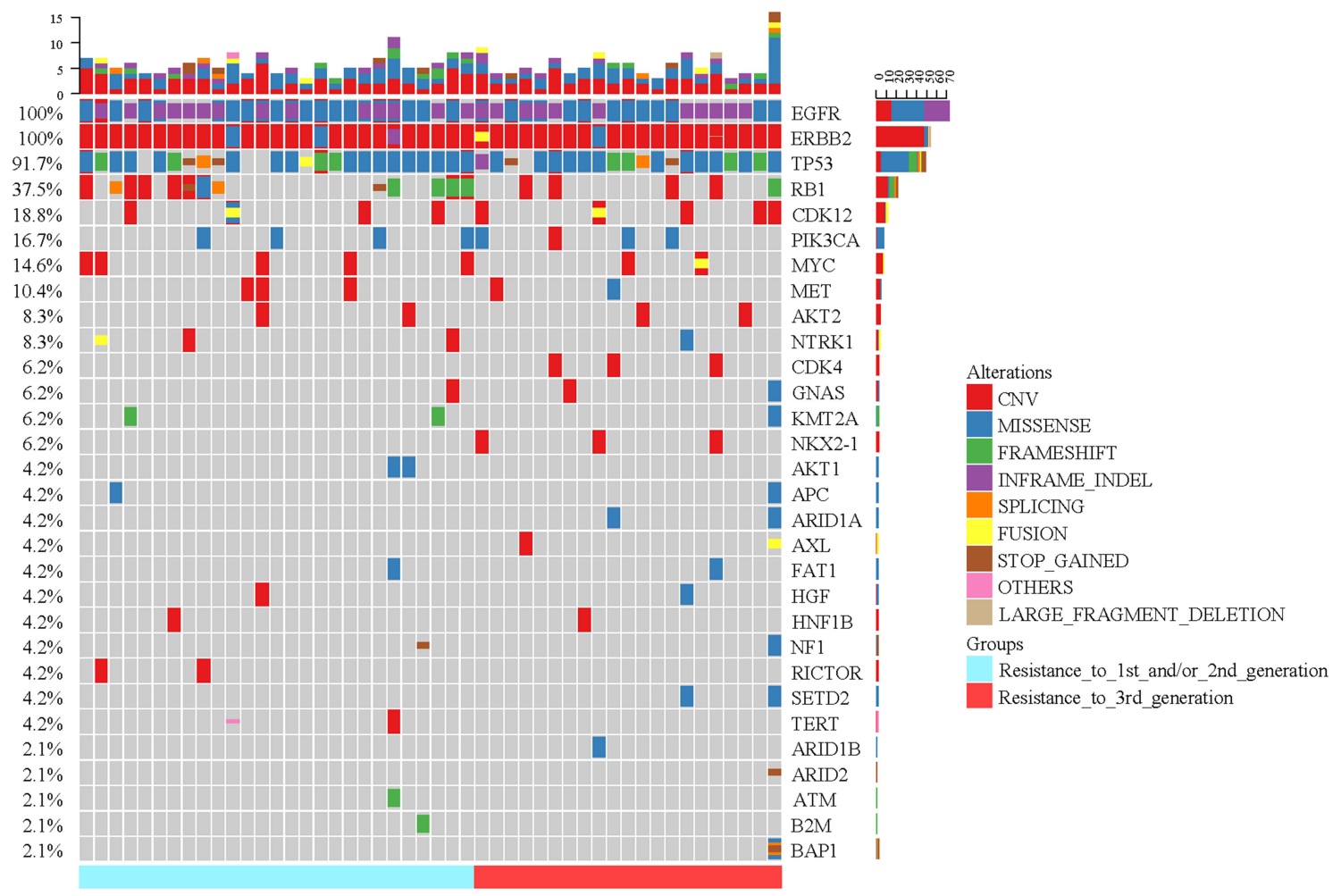

Figure 3 Co-mutation patterns of the 48 patients with concomitant EGFR mutations and HER2 amplification lung cancer from the SYSUCC cohort. Twenty-seven patients were included from progression of I st/2nd generation EGFR-TKI, and $2 \mathrm{I}$ patients were included from progression of 3rd generation. The most frequently mutated gene was TP53 mutation ( $91.7 \%$ patients). The most frequent type of alteration was missense. 


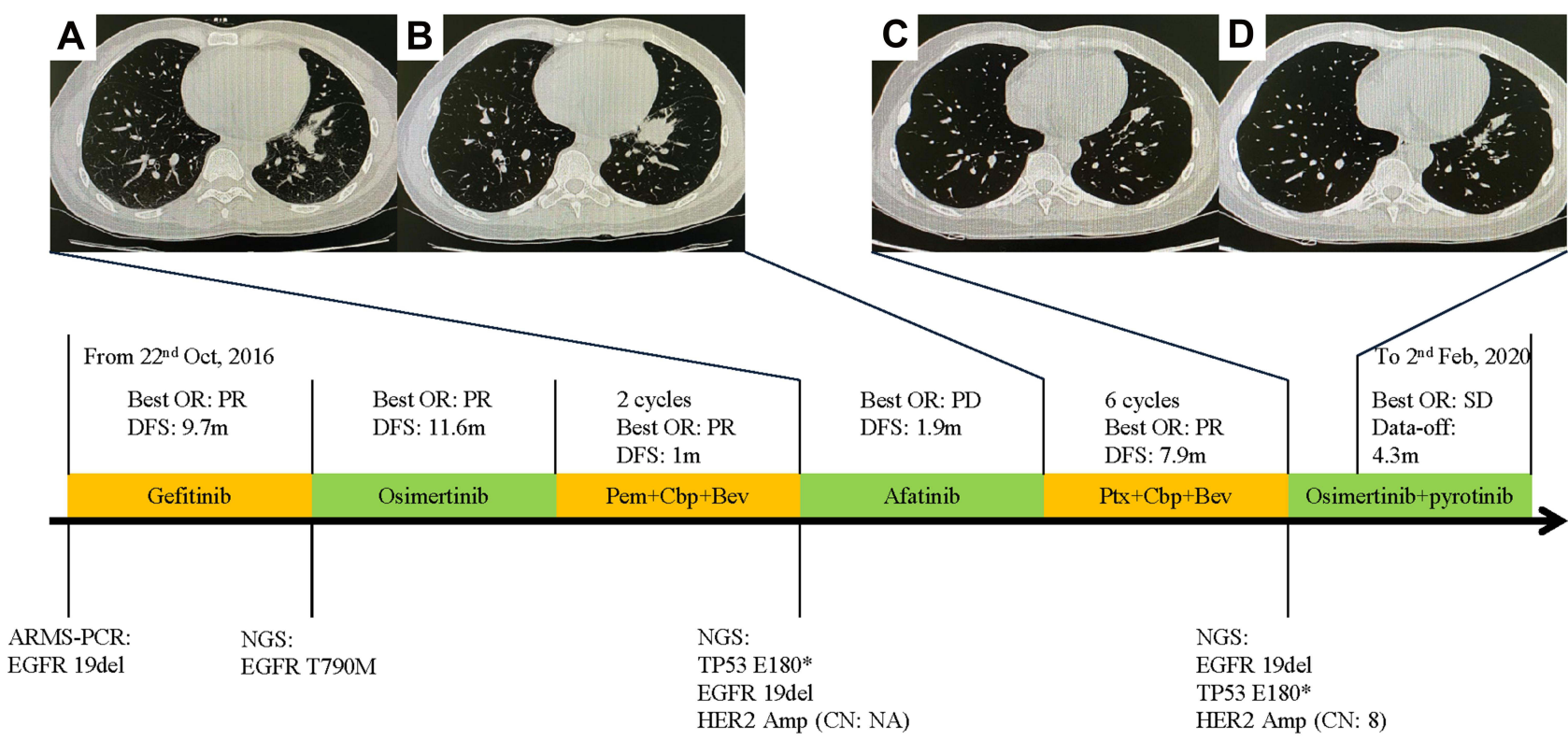

Figure 4 Timeline of treatment course and radiological evaluation before and after Afatinib or combined therapy of EGFR-TKls and pyrotinib. (A) Chest-CT before treatment of Afatinib; (B) Chest-CT after I month treatment of Afatinib; (C) Chest-CT before treatment of osimertinib plus pyrotinib; (D) Chest-CT after I month treatment of osimertinib plus pyrotinib.

Abbreviations: OR, objective response; PR, partial response; PFS, progression-free survival; m, month; PD, progression disease; SD, stable disease; Pem, pemetrexed; Cbp, Carboplatin; Bev, Bevacizumab; amp, amplification; Ptx, paclitaxel.

demonstrated no brain metastasis. Bone emission-CT demonstrated iliac bone metastatis. Pathology revealed adenocarcinoma and ARMS-PCR revealed an EGFR E746_A750del mutation. She was enrolled in a clinical trial and treated with gefitinib and apatinib for 10 months until progression disease. Genomic testing identified EGFR T790M mutation. She then received second-line osimertinib and achieved progression 1 year later. Two cycles of pemetrexed plus carboplatin and bevacizumab were performed and repeat NGS revealed concomitant EGFR E746_A750del and HER2 amplification. Therefore, the patient transformed treatment of afatinib. She experienced diarrhea and rash and the therapeutic effect was evaluated by $\mathrm{CT}$ as progression of disease 1 month later. Osimertinib plus pyrotinib was initiated on September 24, 2019, and evaluation CT of chest demonstrated a best objective response of $\mathrm{SD}$ by data on February 2, 2020. However, brain-MRI revealed the development of new meningeal metastasis, resulting in the evaluation of progressive disease.

\section{Patient 5}

The fifth patient was an 80-year-old man with cough diagnosed with stage IV lung adenocarcinoma with metastases to the brain and liver (Figure 5). NGS analysis of the liver metastases biopsy specimen revealed EGFR L858R mutation. Therefore, the patient was enrolled to a clinical trial and underwent treatment with orally administered gefitinib plus anlotinib. Capture-based targeted sequencing on both re-biopsy sample and plasma cell-free DNA revealed the harboring of EGFR L858R mutation and HER2 amplification after progression. He then switched to gefitinib plus pyrotinib. His clinical symptoms such as cough and dyspnea were remarkably relieved and a radiograph revealed $\mathrm{PR}$ in his lung lesion and the PFS reached 8 months. Chest $\mathrm{CT}$ scan after 8 months showed increasing primary tumor size and fibrous patchy shadows. Genomic analysis on the plasma sample showed EGFR T790M mutation and HER2 amplification. Based on this result, he commenced on osimertinib plus pyrotinib on July 29, 2020 and a CT performed 1 month later demonstrated therapeutic evaluation of SD. Treatment of osimertinib plus pyrotinib was administered until October 29, 2020 .

\section{Discussion}

In this study, 1,637 NSCLC cases from progression of EGFR-TKIs were screened and analyzed for genetic alterations leading to HER2 bypass signaling in resistant EGFR-positive NSCLC by NGS. We detected HER2 amplification in $3 \%$ of $1 \mathrm{st} / 2 \mathrm{nd}$-generation EGFR-TKI and $4 \%$ of 3rd-generation EGFR-TKI (Figure 1). We also 


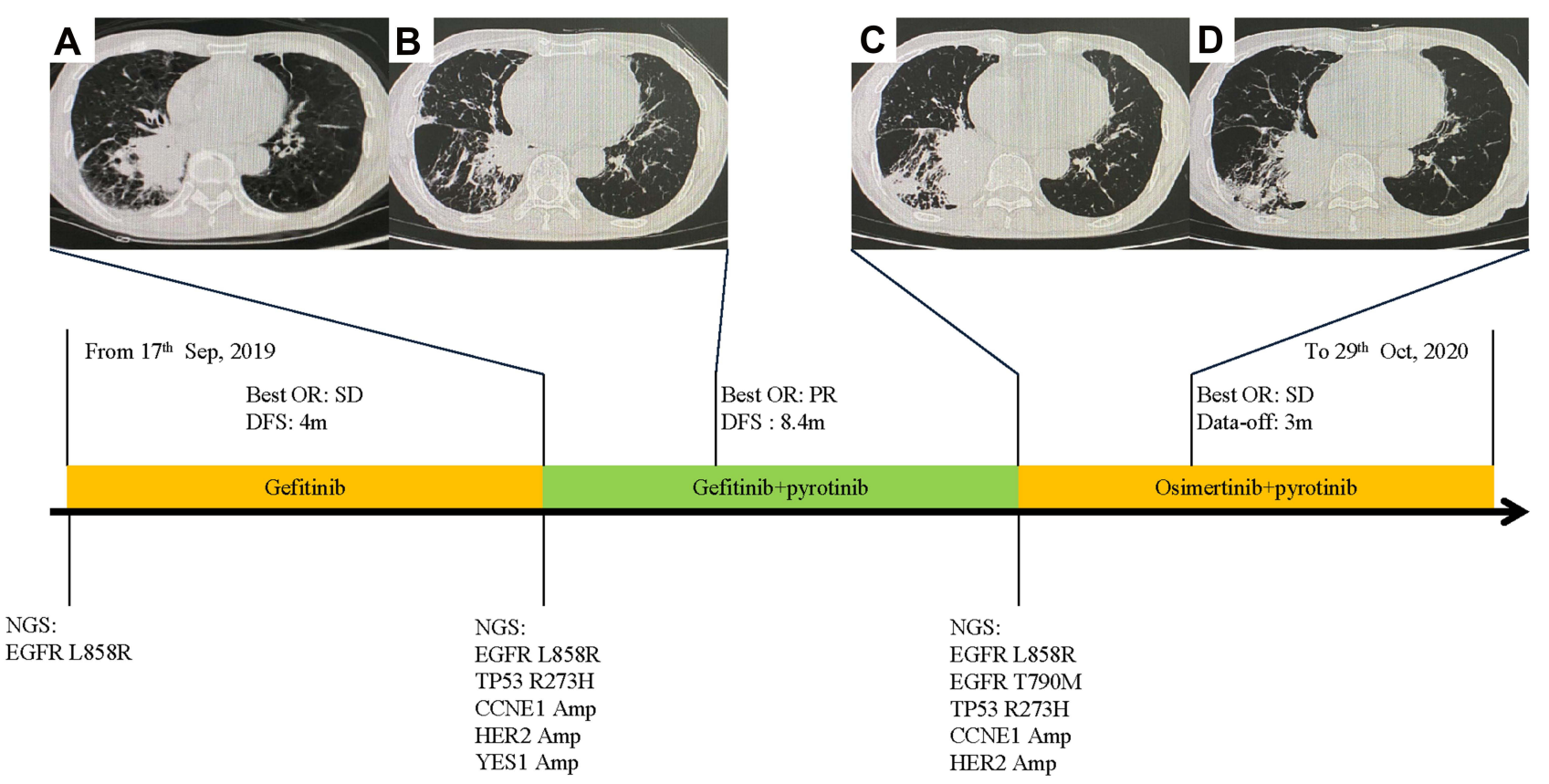

Figure 5 Time line of treatment course and radiological evaluation before and after combined therapy of EGFR-TKIs and pyrotinib. (A) Chest-CT before treatment of gefitinib plus pyrotinib; (B) Chest-CT after 3 months treatment of osimertinib plus pyrotinib; (C) Chest-CT before treatment of osimertinib plus pyrotinib; (D) Chest-CT after I month treatment of osimertinib plus pyrotinib.

Abbreviations: OR, objective response; SD, stable disease; PFS, progression-free survival; m, month; amp, amplification; PR, partial response.

paid attention to the other co-mutations for the enrolled 48 patients after progression of EGFR-TKIs. The common co-occurring alterations were TP53, RB1, CDK12 PIK3CA, and MYC genes (Figure 3). Many of these may serve as bypass resistant mechanisms and are potentially targetable. Consistent with former studies, HER2 mutated patients in our cohort were mainly females with histology of adenocarcinoma lung cancer. ${ }^{22}$

The optimal choice of targeted therapy for HER2 mutation remains poorly defined in lung cancer. HER2-TKIs have primarily shown activity against exon 20 insertion mutations and not so much with the other mutation types.

Afatinib was reported to exhibit varied activity in patients with lung adenocarcinoma carrying HER2 mutations. De Grève et $\mathrm{al}^{23}$ reported in a Phase II study that three (two exon 20 insertion and one point mutation) of five NSCLC patients with bearing HER2 mutations benefit from afatinib. In another phase II clinical trial, ${ }^{24}$ afatinib was reported to have resulted in a $71 \%$ disease control rate (DCR) in patients with HER2 mutation and a certain effect in patients with EGFR amplification. One phase II study of a cohort of 13 patients pretreated for lung adenocarcinoma harboring HER2 exon 20 mutation treated with afatinib monotherapy experienced an objective response rate (ORR) of $7.7 \%(1 / 13){ }^{25}$ Pyrotinib has also been studied in treatment of NSCLC. In one phase II cohort of 15 patients with HER2 exon 20 insertions NSCLC, pyrotinib $400 \mathrm{mg}$ resulted in an ORR of $53.3 \%$ and a PFS of 6.4 months. ${ }^{18}$ Another phase II study demonstrated pyrotinib showed promising antitumor activity with PFS of 6.9 months and overall survival (OS) of 14.4 months in prior chemotherapy-treated patients with HER2-mutant (49 exon 20 insertion and 11 point mutation) NSCLC. Treatment-related adverse events were acceptable, with grade 3 or 4 occurring in $28.3 \%$ of patients. ${ }^{26}$ Kris et al ${ }^{17}$ demonstrated three of 26 patients with tumors harboring HER2 exon 20 mutations (12\%) had PR from dacomitinib monotherapy and fourpatients with HER2 amplification achieved no clinical benefit. A phase II clinical trial (PUMA-NER-4201) evaluating the usefulness of neratinib \pm temsirolimus in HER2 exon 20 insertions NSCLC patients is currently ongoing. ${ }^{16}$ Moreover, co-treatment of anti-HER2 antibodies of trastuzumab or T-DM1 (trastuzumab emtansine) with chemotherapy showed a promising clinical effect in lung cancer patients with HER2 exon 20 insertions obtaining an ORR of $50.9 \% .^{12}$ Phase II clinical trials presented the preliminary efficacy of trastuzumab and TDM-1 may be better than HER2-TKIs in lung cancer. ${ }^{20}$ It may be meaningful to carry out relevant clinical trials in combination of trastuzumab or TDM- 
1 with one mTOR inhibitor such as temsirolimus in the future.

To our knowledge, this is the first description of clinical efficacy of afatinib or combined EGFR-TKIs with pyrotinib on NSCLC harboring EGFR mutation and HER2 amplification simultaneously. We presented three patients treated with afatinib and two with EGFR-TKIs plus pyrotinib. Patient 3 benefited from afatinib in 6 months with a tolerable treatment-related adverse effect. Unfortunately, brain metastasis developed later on and subsequently led to brain failure and follow-up losing, which may be due to the poor ability in penetrating the blood-brain barrier of afatinib. Patient 5 had significant tumor shrinkage with combined treatment of gefitinib and pyrotinib, and achieved a PFS of 8 months. Further studies may try to investigate if genomic determinants of response may differ when monoclonal antibodies were added to EGFR-TKIs. Patients 1 and 2 quickly developed disease progression. Patient 4 achieved the best objective of SD response to osimertinib and pyrotinib for 4 months and he is under continuing treatment without significant symptoms. These cases foreshadow the future of durable and effective clinical management for the subset of patients with concomitant EGFR mutation and HER2 amplification. Combined treatment of EGFR-TKI and pyrotinib acquiring longer survival times may result from inhibiting EGFR and HER2 signaling pathways simultaneously. Furthermore, current clinical studies presented better efficacy of pyrotinib versus afatinib, the curative effect of afatinib remains controversial in lung cancer. $^{20,26}$ Moreover, patients with HER2 alterations may benefit more from standard conventional chemotherapy to targeted therapy, as shown in our listed patients.

Our study does have several limitations. Firstly, it was a retrospective study with limited patients $(n=5)$, while this study presented real world cases in Chinese population. The occurrence of concomitant EGFR mutations and HER2 amplification is rare, and future perspective trials focusing on combined targeted therapy are warranted to provide solid evidence and help in achieving more effective outcomes. Secondly, sample type and panel of the gene-test of the 14 patients we presented varied, which may induce the genetic measuring incomplete and could not show the accurate dynamic changes of the gene alterations. Thirdly, some of the patients were lost to follow-up and the efficacy of the therapeutic regimen could not be better presented and measured.
In this study, we portray the rough genetic landscape of EGFR-TKI resistance. The application of afatinib on NSCLC patients harboring EGFR mutation and HER2 amplification simultaneously needs further clinical evidence. Combination therapies containing EGFR-TKIs and pyrotinib shed some light on potential treatment options for this subset of patients in the future. In conclusion, we roughly explored the treatment strategy of concomitant EGFR mutation and HER2 amplification after EGFR-TKI treatment.

\section{Statement of Consent}

This study was conducted in accordance with the Declaration of Helsinki (as revised in 2013) and approved by Sun Yat-sen University Cancer Center IRB (B2018092-01). We have received the consent to publish the patients' details in this study.

\section{Acknowledgments}

This work was financially supported by the Chinese National Natural Science Foundation Project (81972556, 81772476, and 81872499).

\section{Disclosure}

The authors report no conflicts of interest in this work.

\section{References}

1. Miller KD, Goding Sauer A, Ortiz AP, et al. Cancer statistics for hispanics/ latinos, 2018. CA Cancer J Clin. 2018;68:425-444. doi:10.3322/caac. 21494

2. Planchard D, Boyer MJ, Lee JS, et al. Postprogression outcomes for osimertinib versus standard-of-care EGFR-TKI in patients with previously untreated EGFR-mutated advanced non-small cell lung cancer. Clin Cancer Res. 2019;25(7):2058-2063. doi:10.1158/1078-0432. CCR-18-3325

3. Shaw AT, Kim DW, Nakagawa K, et al. Crizotinib versus chemotherapy in advanced ALK-positive lung cancer. $N$ Engl $J$ Med. 2013;368:2385-2394. doi:10.1056/NEJMoa1214886

4. Shaw AT, Ou SHI, Bang,YJ, et al. Crizotinib in ROS1-rearranged non-small-cell lung cancer. $N$ Engl J Med. 2014;371(21):1963-1971. doi:10.1056/NEJMoa1406766

5. Planchard D, Besse B, Groen HJM, et al. Dabrafenib plus trametinib in patients with previously treated BRAF(V600E)-mutant metastatic non-small cell lung cancer: an open-label, multicentre Phase 2 trial. Lancet Oncol. 2016;17(7):984-993. doi:10.1016/S1470-2045(16) 30146-2

6. Rosell R, Moran T, Queralt C, et al. Screening for epidermal growth factor receptor mutations in lung cancer. $N$ Engl $J$ Med. 2019;361:958-967. doi:10.1056/NEJMoa0904554

7. Shi Y, Au JSK, Thongprasert S, et al. A prospective, molecular epidemiology study of EGFR mutations in asian patients with advanced non-small-cell lung cancer of adenocarcinoma histology (PIONEER). $J$ Thorac Oncol. 2014;9:154-162. doi:10.1097/ JTO.0000000000000033 
8. Recondo G, Facchinetti F, Olaussen KA, Besse B, Friboulet L. Making the first move in EGFR-driven or ALK-driven NSCLC: first-generation or next-generation TKI? Nat Rev Clin Oncol. 2018;15:694-708. doi:10.1038/s41571-018-0081-4

9. Soria JC, Ohe Y, Vansteenkiste J, et al. Osimertinib in untreated EGFR -mutated advanced non-small-cell lung cancer. $N$ Engl J Med. 2018;378:113-125. doi:10.1056/NEJMoa1713137

10. Camidge DR, Pao W, Sequist LV. Acquired resistance to TKIs in solid tumours: learning from lung cancer. Nat Rev Clin Oncol. 2014;11:473-481. doi:10.1038/nrclinonc.2014.104

11. Mar NVJ, Vredenburgh JJ, Wasser,JS. Targeting HER2 in the treatment of non-small cell lung cancer. Lung Cancer. 2015;87 (3):220-225. doi:10.1016/j.lungcan.2014.12.018

12. Mazieres JBF, Barlesi F, Filleron T, et al. Lung cancer patients with HER2 mutations treated with chemotherapy and HER2-targeted drugs: results from the European EUHER2 cohort. Ann Oncol. 2016;27(2):281-286. doi:10.1093/annonc/mdv573

13. Li BT, Ross DS, Aisner,DL, et al. HER2 amplification and HER2 mutation are distinct molecular targets in lung cancers. J Thorac Oncol. 2016;11(3):414 419. doi:10.1016/j.jtho.2015.10.025

14. Takezawa K, Pirazzoli V, Arcila ME, et al. HER2 amplification: a potential mechanism of acquired resistance to EGFR inhibition in EGFR-mutant lung cancers that lack the second-site EGFR T790M mutation. Cancer Discov. 2012;2(10):922-933. doi:10.1158/21598290.CD-12-0108

15. Ettinger DS, Wood DE, Aisner DL, et al. NCCN guidelines insights: non-small cell lung cancer, version 2.2021: featured updates to the NCCN guidelines. J Natl Compr Canc Netw. 2021;19:254-266. doi:10.6004/jncen.2021.0013

16. Gandhi L, Besse B, Mazieres J, et al. MA04.02 neratinib \pm temsirolimus in HER2-mutant lung cancers: an international, randomized phase II study. $J$ Thoracic Oncol. 2017;12(1):S358-S359. doi:10.1016/j.jtho.2016.11.398

17. Kris MG, Camidge DR, Giaccone G, et al. Targeting HER2 aberrations as actionable drivers in lung cancers: phase II trial of the pan-HER tyrosine kinase inhibitor dacomitinib in patients with HER2-mutant or amplified tumors. Ann Oncol. 2015;26 (7):1421-1427. doi:10.1093/annonc/mdv186
18. Wang Y, Jiang T, Qin Z, et al. HER2 exon 20 insertions in non-smallcell lung cancer are sensitive to the irreversible pan-HER receptor tyrosine kinase inhibitor pyrotinib. Ann Oncol. 2019;30(3):447-455. doi:10.1093/annonc/mdy542

19. Ramlau R, Thomas M, Novello S, et al. Phase I study of lapatinib and pemetrexed in the second-line treatment of advanced or metastatic non-small-cell lung cancer with assessment of circulating cell free thymidylate synthase RNA as a potential biomarker. Clin Lung Cancer. 2015;16(5):348-357. doi:10.1016/j.cllc.2015.01.004

20. Zhao J, Xia Y. Targeting HER2 alterations in non-small-cell lung cancer: a comprehensive review. JCO Precis Oncol. 2020;3 (4):411-425. doi:10.1200/PO.19.00333

21. Eisenhauer EA, Therasse P, Bogaerts J, et al. New response evaluation criteria in solid tumours: revised RECIST guideline (version 1.1). Eur $J$ Cancer. 2009;45(2):228-247. doi:10.1016/j. ejca.2008.10.026

22. Arcila ME, Chaft JE, Nafa K, et al. Prevalence, clinicopathologic associations, and molecular spectrum of ERBB2 (HER2) tyrosine kinase mutations in lung adenocarcinomas. Clin Cancer Res. 2012;18(18):4910-4918. doi:10.1158/1078-0432.CCR-12-0912

23. De Grève J, Teugels E, Geers C, et al. Clinical activity of afatinib (BIBW 2992) in patients with lung adenocarcinoma with mutations in the kinase domain of HER2/neu. Lung Cancer. 2012;76 (1):123-127. doi:10.1016/j.lungcan.2012.01.008

24. De Grève J, Moran T, Graas,MP, et al. Phase II study of afatinib, an irreversible ErbB family blocker, in demographically and genotypically defined lung adenocarcinoma. Lung Cancer. 2015;88(1):63-69. doi:10.1016/j.lungcan.2015.01.013

25. Smit EF, Peters S, Dziadziuszko R, et al. A single-arm phase II trial of afatinib in pretreated patients with advanced NSCLC harboring a HER2 mutation: the ETOP NICHE trial. J Clin Oncol. 2017;35 (15_suppl). doi:10.1200/JCO.2017.35.15_suppl.9070

26. Zhou C, Li X, Wang Q, et al. Pyrotinib in HER2-mutant advanced lung adenocarcinoma after platinum-based chemotherapy: a multicenter, open-label, single-arm, phase II study. J Clin Oncol. 2020;38(24):2753-2761. doi:10.1200/JCO.20.00297
OncoTargets and Therapy

\section{Publish your work in this journal}

OncoTargets and Therapy is an international, peer-reviewed, open access journal focusing on the pathological basis of all cancers, potential targets for therapy and treatment protocols employed to improve the management of cancer patients. The journal also focuses on the impact of management programs and new therapeutic agents and protocols on patient perspectives such as quality of life, adherence and satisfaction. The manuscript management system is completely online and includes a very quick and fair peer-review system, which is all easy to use. Visit http://www.dovepress.com/ testimonials.php to read real quotes from published authors. 